\title{
Análisis de las prácticas socio comunitarias en el marco de una institución pública de educación superior: Compromiso social y potencialidad transformadora
}

\author{
Romina TIRITILLI, Paola GIOMETTI, Wanda BOSCH, Yamila SILVA-PERALTA ${ }^{1}$ y \\ Miriam T. APARICIO
}

Universidad Mar de la Plata, Argentina

(Recibido 27 Febrero, 2016; Aceptado 14 Junio, 2016)

RESUMEN: La presente investigación pretende indagar los aspectos transformadores de las prácticas socio comunitarias (PSC) realizadas por estudiantes avanzados de una institución pública de educación superior. Se trata de una investigación exploratoria descriptiva y cualitativa en la que se utilizaron diferentes instrumentos: observación no participante del dispositivo de tutorías a lo largo del dictado de las prácticas; observación no participante de los coloquios finales expuestos por los grupos de estudiantes; entrevistas de incidentes críticos a docentes tutores; y análisis de los informes escritos finales realizados por los estudiantes. La muestra fue intencional compuesta por estudiantes $(\mathrm{N}=65)$ y docentes tutores $(\mathrm{N}=6)$ de las PSC de una institución pública de educación superior. Se calcularon descriptivos básicos y se realizó análisis de contenido de los incidentes críticos y de los informes escritos presentados. Los resultados muestran que la realización de las PSC, a través de la metodología de Aprendizaje Servicio (APS), promueve la transformación de los estudiantes en las dimensiones epistemológica, social, pedagógica y ética. Estas transformaciones promueven la aparición de conductas prosociales favoreciendo el desarrollo ciudadano, un posicionamiento ético en la actividad profesional, la reflexión crítica y el compromiso con la comunidad.

Palabras clave: Compromiso social universitario, aprendizaje servicio, universidad.

Socio community practices' analyses at a public superior education institution: Social commitment and transformational potential

ABSTRACT: The present research pretends to know about socio community practices' transformational aspects done by advanced students from a superior education public institution. It's an exploratory descriptive and qualitative research in which there were used different instruments: tutorials non participant observation during the practices; final students' colloquies non participant observation; teachers' critical incidents interviews and students' final written reports. The sample was intentional composed by students $(\mathrm{N}=65)$ and teachers $(\mathrm{N}=6)$ from a superior education public institution socio community practices. Basics descriptive statistics were calculated and critical incidents' and final written reports' content analyses were done. Results show that socio community practices following the social learning technique promote students transformation in epistemology, social, pedagogic and ethic dimensions. These transformations promote pro social and citizenship 
behaviors, an ethic position related to professional activity, a critical reflection and community commitment.

Keywords: University social commitment; service-learning, university.

${ }^{1}$ Correspondencia: Silva Peralta, Yamila, c/ Funes 3250 (7600) Mar del Plata, Buenos Aires, Argentina. E-mail: yamilasilvaperalta@gmail.com.

\section{Prácticas socio comunitarias y compromiso social universitario}

Durante los últimos treinta años se han producido transformaciones sociales, económicas y culturales que han puesto de relieve la necesidad de repensar las implicancias que la educación superior tiene en el desarrollo de las personas y de las sociedades en su conjunto. Como es señalado en el preámbulo de la Declaración de la Conferencia Mundial sobre la Educación Superior del año 2009 (p.1), nunca antes en la historia fue más importante la inversión en educación superior en tanto ésta constituye una base fundamental para la construcción de una sociedad del conocimiento inclusiva y diversa, y para el progreso de la investigación, la innovación y la creatividad.

Gasca-Pliego y Olvera-García (2011) sostienen que la educación superior debe mantenerse actualizada respecto de los problemas de la agenda de desarrollo local y global generando estrategias de intervención pedagógica e investigación aplicada, consecuentes con esas problemáticas. La Universidad actual comienza a reconsiderar su misión en términos de pertinencia social (Muñoz, 2008), asumiendo su rol fundamental en la solución de las problemáticas sociales así como impulsando el fortalecimiento de la cohesión social. Para cumplir con estos nuevos desafíos y posibilidades de acción de la universidad, es menester asumir un rol responsable frente a las necesidades de la sociedad. La Responsabilidad Social Universitaria (RSU), concepto heredado y transformado desde el mundo de la empresa, debe entenderse, según De la Cuesta González, De la Cruz Ayuso y Fernández (2010), como el compromiso implícito en la naturaleza misma de la Universidad de difundir y poner en práctica un conjunto de principios y valores generales y específicos, por medio de los procesos de gestión, docencia, investigación y extensión. Mazzetti (2014) en su revisión teórica sobre el tema, sostiene que es la noción de Compromiso Social Universitario (CSU) la que se ajusta a este desafío de la educación superior, y lo define como el compromiso que debe ejercer la universidad, teniendo en cuenta su misión social en cada una de sus prácticas singulares arraigadas curricularmente. Según García Guadilla (2008) el CSU permite la formación de nuevos profesionales y científicos comprometidos con la comunidad y con conciencia social.

Muñoz (2008) sostiene que uno de los criterios posibles para evaluar la calidad de la educación superior es el de la pertinencia social, laboral, cultural y ecológica, que sólo podrá desarrollarse de manera efectiva, si y sólo si, en los planes de formación y estructuras académicas se promueve la flexibilidad e interdisciplinariedad necesarias para posibilitar la adaptación a los vertiginosos cambios experimentados en las profesiones, la ciencia y la vida social.

Una de las tendencias más transformadoras que se han producido en algunas de las universidades argentinas en los últimos años, radica en la formalización de las PSC, fundándose sobre experiencias anteriores de otras universidades de la región como las de (c) Psy, Soc, \& Educ, 2016, Vol. 8(3) 
México, Costa Rica y Venezuela. En algunos casos, estas PSC se encuentran curricularizadas en los planes de estudio de instituciones de educación superior. Según Cecchi, Delicio, Governatori, Libera, Puglisi y Zaballa (2010), las PSC proponen que el estudiante desarrolle valores solidarios, el compromiso social, y un posicionamiento ético y crítico que trace un puente entre la Universidad y la comunidad. En este sentido Cecchi y Pérez (2013) señalan que las PSC promueven transformaciones en las dimensiones ética, epistemológica, social y pedagógica.

Estas propuestas de PSC han construido sus estrategias didácticas sobre la base de un nuevo modelo de aprendizaje: la metodología del Aprendizaje Servicio (APS), definida como el servicio solidario desarrollado por los estudiantes, destinado a cubrir necesidades reales de una comunidad, planificado institucionalmente en forma integrada con el currículum, en función del aprendizaje de los estudiantes (Tapia, 2002, p. 3). En los años '90s esta metodología era prácticamente desconocida y desde entonces el crecimiento ha sido evidente ya que, según Tapia (2002), es practicada por lo menos por 5.000 escuelas (aproximadamente $13 \%$ del total) y cerca de un centenar de universidades. Según la autora, el APS consta de tres notas características: (a) está protagonizado fundamentalmente por los estudiantes, (b) se propone atender solidariamente una necesidad real y sentida por la comunidad, y (c) está planificada no sólo para atender a esa necesidad social, sino para mejorar la calidad de los aprendizajes escolares.

La pedagogía del APS comienza a conceptualizarse a fines de la década del'60, contemporáneamente al período en que convergen la tradición norteamericana de experiential learning y la experiencia latinoamericana, liderada por Paulo Freire, con su propuesta de Pedagogía Critica. Con su publicación en inglés de Pedagogía del oprimido (1970), sus conceptos fueron difundidos en ámbitos académicos de todo el mundo. Tal como lo menciona Tapia, aún hoy el pensamiento de Paulo Freire - incluida su Pedagogía de la esperanza-, puede reconocerse en los fundamentos teóricos tanto del aprendizaje-servicio latinoamericano como en el service-learning norteamericano (Tapia, 2010, p. 31).

Según Campo (2008), cuando un estudiante vive intensamente una experiencia de APS, no vuelve a mirar sus estudios ni su profesión de la misma manera, ya que su formación teórico técnica ha sido atravesada por la vivencia del territorio y de toda su complejidad. Esta experiencia metodológica permite al estudiante, no sólo crecer como persona, sino ampliar su mirada social y profesional dando significado a conocimientos académicos, sensibilizándose mediante la vivencia de valores como la solidaridad, el respeto y el compromiso.

\section{Evaluación de acciones formativas: Modelo de Kirkpatrick}

La evaluación de la formación en las organizaciones es una estrategia necesaria para garantizar la calidad de las acciones formativas que se llevan a cabo en su seno y para impulsar el proceso de aprendizaje constante que el entorno actual exige.

Guerra-López (2007) plantea que la evaluación de la formación/capacitación tiene en los años 60s una importancia económica en Norteamérica dadas las cuantiosas inversiones que se realizaban en programas socio-educativos y de salud. Es recién en los 70s y 80s que este concepto trasciende lo gubernamental para pasar a cobrar relevancia en el ámbito privado. Mediante la evaluación de la capacitación compara resultados respecto a expectativas, 
encuentra los conductores apropiados y las barreras respecto al desempeño esperado; $y$ produce planes de acción para mejorar programas y soluciones que están siendo evaluadas para poder lograr y/o mantener el desempeño esperado para que los objetivos $y$ contribuciones organizacionales puedan ser cumplidos (p.11).

Los modelos más citados de evaluación de la formación/capacitación son (Rutty, 2007): el modelo de Kirkpatrick (1999) elegido en esta investigación porque estableció las bases de la evaluación de la formación continua, será desarrollado más adelante. El modelo ROI de Jack Phillips, más cuantitativo, agrega un quinto nivel al modelo anterior centrado en medir el impacto económico de la capacitación en una empresa a través del cálculo de retorno de inversión (ROI) (Guerra-López, 2007). Como desventaja, podría decirse que no todo proyecto de capacitación es adecuado para evaluar ROI ya que aquellos que están ligados a una estrategia de la empresa, suelen ser a largo plazo (Olea Soto, 2010, p.20). Finalmente, el modelo HPI o Human Performance Improvement, que enfatiza en las oportunidades de mejora continua en el desempeño/rendimiento en el puesto de trabajo (Rothwell, Hohne \& King, 2007), entonces, se concentra sobre todo en el tercer nivel de Kirkpatrick.

A continuación detallaremos los niveles que propone el modelo elegido.

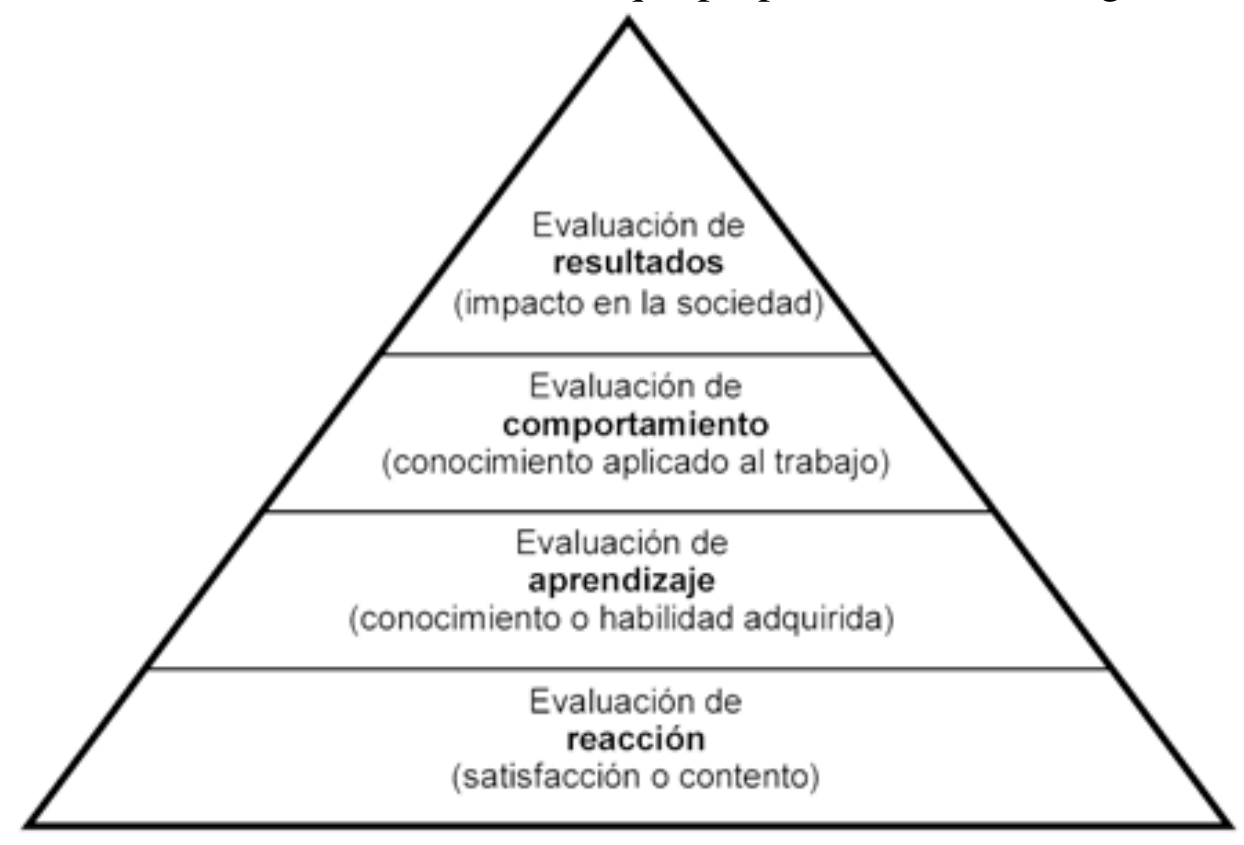

Figura 1. Modelo de evaluación de acciones formativas de Kirkpatrick (1999).

El nivel de Reacciones mide la cuota de satisfacción de los estudiantes respecto de la formación recibida y la valoración positiva/negativa de los diversos atributos del curso de formación. Una satisfacción positiva favorece la permanencia de la actuación formativa. El segundo nivel de Aprendizaje normalmente es utilizado para realizar una medición efectiva de los aprendizajes adquiridos y pretende dar cuenta de las transformaciones ocurridas en las competencias personales. Con motivo de este trabajo se pretende evaluar el aprendizaje en términos de desarrollo y adquisición de competencias según el Modelo de Competencias de Acción Profesional de Bunk (1994). Este último presenta competencias en cuatro niveles: competencia técnica: refiere al dominio como experto de las tareas y contenidos del ámbito de trabajo, así como los conocimientos y destrezas necesarios para ello; competencia 
metodológica: refiere a la capacidad de reaccionar aplicando el procedimiento adecuado, encontrar soluciones y transferir experiencias; competencia social: refiere a la colaboración con otras personas de forma comunicativa y constructiva mostrando un comportamiento orientado al grupo y un entendimiento interpersonal; competencia participativa: refiere a la capacidad de organizar y decidir aceptando responsabilidades, se amplía el sentido de la participación en este nivel de análisis para incluir su carácter político aportando atributos transformadores como el compromiso ético, la valoración de la interdisciplina, la valoración de la responsabilidad social y el desarrollo de un sentido crítico que valore la participación de todos los actores. El tercer nivel del modelo de Kirkpatrick llamado Transferencia, pretende medir si los estudiantes pueden aplicar en su trabajo/práctica profesional los conocimientos adquiridos. Finalmente, el nivel de Impacto Organizacional evalúa si los objetivos planteados en la acción formativa se utilizan en la organización de forma efectiva y eficiente.

Las ventajas del modelo de Kirkpatrick son su viabilidad práctica o aplicabilidad según formadores y educadores. Como críticas, este modelo ha sido considerado simplista y algunos autores plantean que se estanca en el segundo nivel de análisis (aprendizaje), así como los tiempos requeridos para evaluar feedback y asimilación (Olea Soto, 2010). Sin embargo, a los fines de esta investigación es válida su estructura en cuatro niveles ya que se alinea con las dimensiones de evaluación pedagógica que propone el seminario de prácticas comunitarias analizado en la presente.

\section{Método}

Se trata de un estudio exploratorio-descriptivo realizado sobre las PSC de una institución pública de educación superior. El objetivo general fue el de explorar y describir las prácticas en relación a situaciones que puedan ser evaluadas como transformadoras por docentes tutores y estudiantes participantes, teniendo en cuenta el modelo de evaluación de Kirkpatrick (1999) previamente presentado.

Para alcanzar los objetivos estipulados se propuso analizar: (1) el dispositivo de tutorías de las prácticas mediante la observación no participante, (2) los impactos de las PSC explicitados por los estudiantes de acuerdo a las dimensiones de análisis propuestas (académica, profesional, comunitaria y personal) mediante el análisis documental y la observación no participante de los coloquios de los trabajos finales, y (3) situaciones críticas consideradas por docentes como favorables y no favorables mediante entrevistas de incidentes críticos siguiendo la técnica de Flanagan (1954). Según su inventor, la Técnica del Incidente Crítico es un conjunto de procedimientos que permiten reunir observaciones directas del comportamiento humano, facilitando así su uso potencial para resolver problemas prácticos y desarrollar principios psicológicos amplios. Por otro lado, sugiere que debe ser entendida como un conjunto flexible de principios que deben ser modificados y adaptados para satisfacer la situación específica que se tiene a la mano.

Posteriormente, esta técnica se extiende a otros ámbitos como el educacional; un ejemplo de ello es trabajo de Nail (2010), quien sigue los desarrollos de David Tripp (1993), autor que enfatiza en la potencia de un incidente crítico para la reflexión sobre la convivencia áulica. En la presente investigación se decidió aplicar la técnica siguiendo a su fundador, dado que se indagaron situaciones áulicas así como situaciones extra-áulicas, ya que las PSC 
analizadas trascienden el espacio de la sala de clases para vincularse con la comunidad a través de las diferentes organizaciones locales en las que se desempeñan los estudiantes.

En la presente investigación se observaron 18 clases de tutoría, se realizaron 6 entrevistas semidirigidas a los docentes de la cátedra y se analizaron 32 informes finales grupales correspondientes a 65 estudiantes asistentes a las PSC en el año 2014. Se analizaron las observaciones, entrevistas e informes siguiendo la técnica de análisis de contenido (Navarro \& Díaz, 1994).

La técnica de análisis de contenido, de relevancia significativa en la investigación cualitativa, puede ser considerada como una forma particular de análisis de documentos, que no pretende analizar el estilo del texto, sino las ideas expresadas en él, en tanto que esfuerzo de interpretación (Almenara \& Abril, 2002).

En cuanto al proceso de análisis, el mismo se organizó en tres etapas. En primer lugar se registraron las observaciones de los espacios de tutoría, teniendo en cuenta un instrumento de evaluación que contenía categorías de análisis construidas previamente, como así también un espacio que permitiera la apertura de nuevas categorías. Se registraron citas textuales de las reflexiones de estudiantes y docentes sobre la experiencia vivencial de la PSC, así como cuestiones referidas a la dinámica de trabajo y la propuesta pedagógica de la cátedra.

Una vez concluida la etapa de observación, se procedió a realizar las entrevistas de incidentes críticos a los docentes de la cátedra, para lo cual se elaboró un instrumento que integrara información proveniente del curriculum de cada docente, la historia de la cátedra, la contextualización en el marco de la institución, la aplicación de la técnica de incidentes propiamente dicha, y la reflexión sobre la propia práctica.

Por último se analizaron los documentos elaborados por los propios estudiantes a la luz de las categorías provistas por el modelo de Kirkpatrick y su integración con el Modelo de Competencias de Acción Profesional (Bunk, 1994). Para el análisis de contenido se construyeron categorías empíricas a través del análisis de citas textuales de estudiantes, detectando la utilización de expresiones comunes que pudieran ser agrupadas bajo categorías más amplias.

Una vez realizado este análisis, se procedió a valorar la recurrencia de contenidos dentro de los niveles de análisis del modelo de Kirkpatrick, y desarrollar así algunas conclusiones sobre los datos obtenidos, refiriendo en forma constante al discurso textual de los participantes para contextualizar los resultados.

\section{Resultados}

Estas PSC se iniciaron en la institución mediante una propuesta realizada por estudiantes. Orientada a generar las transformaciones necesarias en la formación de los profesionales, se propuso lograr una mayor pertinencia de los contenidos y un cumplimiento efectivo de la CSU, como así también un acercamiento al trabajo comunitario por parte de los estudiantes.

Las PSC se articulan en dos fases de trabajo: la primera, de carácter teórico, prepara a los estudiantes para la salida al campo mediante la ampliación de conocimientos específicos técnicos y conceptuales referidos al trabajo comunitario. La segunda fase consiste en el trabajo con las organizaciones y se realiza en pequeños grupos/duplas de estudiantes, en ocasiones 
pertenecientes a diversas carreras, que deben cumplimentar 30 (treinta) horas de trabajo en la comunidad aplicando saberes teórico-técnicos específicos de su formación. Durante esta segunda fase se crea un dispositivo de tutoría que busca propiciar la reflexión y abordar los diversos emergentes de la práctica en territorio. Es un espacio de acompañamiento y evaluación permanente del proceso, que concluye con la presentación de un informe final y un coloquio donde se expone grupalmente lo más relevante de la experiencia.

Estas PSC, según sus propios docentes tutores, se proponen promover transformaciones en los estudiantes en las siguientes dimensiones: ética, epistemológica, social y pedagógica.

A continuación, presentamos el análisis de los resultados según sea la evaluación realizada por los estudiantes o por los docentes tutores de las PSC.

Evaluación realizada por los estudiantes

De acuerdo al modelo de evaluación de acciones formativas de Kirkpatrick (1999) se presentan los resultados agrupados en los cuatro niveles de impacto: Reacciones, Aprendizaje, Transferencia e Impacto Organizacional.

\section{Reacciones:}

En este nivel se mide la cuota de satisfacción de los estudiantes respecto de la formación recibida, así como la valoración positiva y negativa de los diversos atributos del curso de formación. El 95\% de los estudiantes valora positivamente la experiencia, atribuyendo dicha satisfacción a diversos factores que han sido agrupados en tres grandes categorías empíricas, construidas en relación a las vivencias relatadas por los estudiantes. La mayoría de las respuestas se agrupan en la categoría Sentimientos de comunidad, que refiere a la vivencia comunitaria sentida durante el proceso de formación, el trabajo en equipo y las relaciones interpersonales (e.g., "fuimos recibidos con los brazos abiertos, nos hicieron un lugar en su mesa, charlamos y formamos parte de esa pequeña comunidad"). En segundo término, las respuestas se concentraron en la categoría Sentimientos positivos hacia sí mismos y vivencia de auto-superación, indicando que la formación ha colaborado en el crecimiento personal de los estudiantes, devolviéndoles una autopercepción más positiva de sí mismos (e.g., "la experiencia de trabajar con una organización sin fines de lucro fue muy enriquecedora tanto en lo académico, profesional y en lo social, nos permitió llegar a otros sectores de la sociedad y ayudarlos brindándoles nuestros conocimientos, tiempo y colaboración”). Finalmente, un grupo más reducido de estudiantes indican que su satisfacción se atribuye al cumplimiento de los Resultados y objetivos planteados al inicio del proyecto (e.g., "para nosotros, fue una experiencia valiosa ya que logramos todos los objetivos propuestos, sintiéndonos útiles y valorados por la institución”).

2. Aprendizaje:

Este nivel pretende dar cuenta de las transformaciones ocurridas en las competencias personales, en todas sus dimensiones: conocimientos, actitudes, habilidades, dependiendo de los objetivos y naturaleza de la formación. Para ello se han categorizado las respuestas teniendo en cuenta un esquema adaptado al Modelo de Competencias de Acción Profesional (Bunk, 1994). Este último presenta competencias en cuatro niveles: técnicas, metodológicas, sociales y participativas ya desarrolladas en el apartado de Revisión teórica de la presente investigación. A propósito de este análisis se prescindió de evaluar las competencias técnicas, teniendo en cuenta que los conocimientos técnicos ya han sido adquiridos durante toda la formación precedente en la carrera de grado. Luego del análisis, las respuestas indican que el aprendizaje 
se encuentra concentrado en la competencia metodológica, haciendo referencia a la capacidad de reaccionar aplicando el procedimiento adecuado, de encontrar soluciones y transferir experiencias de manera flexible y adaptándose a diferentes contextos (e.g., "por primera vez, nos encontramos parados frente a la realidad, plasmando saberes que entendíamos desde la teoría pero nunca representados en casos concretos y reales, con la responsabilidad que esto conlleva"). La misma importancia fue atribuida a los aprendizajes de la competencia participativa, refiriendo a atributos transformadores como el compromiso ético, la valoración de la interdisciplina, la responsabilidad social y el desarrollo de un sentido crítico que potencie la participación de todos los actores (e.g., "también aprendimos cómo vamos a poder colaborar con la sociedad desde nuestra futura profesión, mirada que no habíamos alcanzado antes además de poder entender la realidad de las mujeres del taller desde otro punto de vista, desde el psicológico, mediante las interesantes charlas que teníamos con la organización”).

En menor medida, se encontraron respuestas referidas al aprendizaje de la Competencia social, poniendo énfasis en la colaboración con otras personas de forma comunicativa y constructiva mostrando un comportamiento orientado al grupo $\mathrm{y}$ un entendimiento interpersonal (e.g., "trabajar con personas en estas condiciones nos revela la importancia de ser creativos y de diseñar nuevos servicios adaptados a las necesidades y capacidades de cada grupo, poniéndonos en su lugar y acompañándolos en el proceso de cambio”).

En la investigación, un grupo reducido de estudiantes valora negativamente la formación recibida para hacer frente de manera solvente a la experiencia de la PSC (e.g., "en la facultad nos preparan principalmente para brindar servicios en organizaciones con fines de lucro y gracias a estas prácticas uno puede tomar contacto con otros sectores de la sociedad que requieren de ayuda profesional”).

3. Transferencia:

Este tercer nivel pretende medir las valoraciones realizadas por los estudiantes acerca de la vivencia del ejercicio del rol profesional durante la práctica y la posibilidad de transferir esta experiencia en su futuro desarrollo profesional. Se construyeron para ello cuatro categorías empíricas de análisis, ellas son: (3.1.) Polivalencia del rol: en esta categoría se agrupan las respuestas que refieren a los diferentes discursos que encontraron en la comunidad respecto del rol profesional: "el profesional es el que sabe”, el profesional como técnico, como consultor, como colaborador. (e.g., "aprendimos que un buen profesional es aquel que es excelente desde el punto de vista técnico, en lo que atañe a los conocimientos, como así también desde el punto de vista social, en lo que hace a los valores como personas, con conciencia de las obligaciones con la comunidad en la que vivimos y de la que venimos”). En similar medida, la PSC permitió a los estudiantes conocer en territorio las Incumbencias profesionales, descubriendo nuevos y diversos ámbitos de trabajo. (e.g., "gracias a la práctica realizada pudimos ampliar nuestro panorama y conocer otro ámbito en el cual es posible desarrollar la profesión y brindar nuestros servicios. ONG, Sociedades de Fomento, comedores barriales, emprendedores y no sólo limitarnos a comercios, empresas, industrias, etc.”). Los estudiantes mencionaron con similar importancia la necesidad de incluir en la labor profesional la cuestión del Compromiso ético del rol, luego de encontrarse con profesionales cuyas conductas observadas en la práctica se alejan de las normas éticas y deontológicas. (e.g., "a la hora de asumir un trabajo de cualquier índole, un profesional debe cumplir con lo acordado o abstenerse en el caso de no sentirse capacitado 
para brindar un mejor servicio al cliente. Nos hemos comprometido en ayudarlos sobre lo que esté a nuestro alcance y asumir la responsabilidad de los trabajos realizados”).

\section{Impacto Organizacional:}

En este último nivel se intenta medir las valoraciones que los estudiantes realizaron respecto del impacto que ha tenido su tarea en la organización en la que realizaron la PSC. Su objetivo es evaluar el beneficio que ha producido la acción formativa en la comunidad. Para el análisis se han dividido las respuestas teniendo en cuenta según dónde los estudiantes valoran que se ha producido el impacto. Se presentan a continuación de acuerdo a su relevancia en los resultados:

En las personas: (e.g., “durante nuestra intervención comenzaron a estar más atentos y a poner mayor esfuerzo por aprender principalmente respecto a las obligaciones de formalización de la organización”).

En los procesos: (e.g., "aprendimos a delegar las responsabilidades y funciones de la organización, a obtener y administrar los recursos y la importancia de tener todo en regla para lograr obtener ayuda de diferentes lugares puesto que la transparencia es vital para la confianza").

En los resultados: (e.g., "nuestra tarea en la institución permitió un ahorro de tiempo y disminución de errores a la hora de realizar la liquidación de sueldos, lo que permite que las encargadas de realizar esa tarea, puedan realizar otro tipo de actividades que se venían postergado").

En la Universidad: (e.g., "al cursar el seminario y realizar las prácticas pudimos dimensionar otras funciones de la universidad, que muchas veces se encuentran relegadas. Comprendimos que la formación tiene que abarcar diferentes aspectos, no sólo el académico o profesional. Tiene que ir más allá, insertarse en el ámbito, preocuparse por el contexto, generar compromiso y propugnar por el cambio. Es una práctica basada en valores (como solidaridad, fraternidad e igualdad). Al hacer la práctica no sólo transformamos la comunidad, sino también nuestra Facultad, cumpliendo con su misión social”).

A su vez se encontraron respuestas que no daban cuenta del impacto en la organización, o bien no especificaban a qué atribuían el mismo.

Evaluación realizada por los docentes

A continuación se presentan los resultados del análisis realizado sobre las entrevistas administradas a docentes mediante la técnica de incidentes críticos (Flanagan, 1954). Según Navarro, López y Barroso (1998) los incidentes críticos son situaciones significativas que ponen al docente en una situación desestabilizante impactando en los planos cognitivo, social y emocional.

Los incidentes evaluados como positivos de los 6 docentes tutores entrevistados se relacionan en la totalidad de los casos con situaciones en que queda en evidencia el compromiso personal e implicación emocional de los estudiantes. Los docentes valoran de manera significativamente satisfactoria que los estudiantes se impliquen en su práctica y que esta sea una primera experiencia de trabajo comunitario, a continuar en el futuro (e.g., "una de las que más me llamó la atención fue la de una estudiante de Pinamar que nos pidió si podía hacer su trabajo allá. Y lo hizo en el cuartel de bomberos voluntarios y fue tan interesante su trabajo, que la terminaron contratando"). 
En cuanto a los incidentes críticos asociados a eventos calificados negativamente, la mayoría de los docentes tutores coinciden en atribuir esa evaluación a los casos de prácticas que ellos denominan de "baja intensidad” (e.g., prácticas en que los estudiantes "van poco a la institución y cuando vienen a la tutoría intentan hacernos creer que han hecho grandes cosas (...) nos damos cuenta cuando no es así”).

Otro de los aspectos indagados en las entrevistas se vincula a las consideraciones que hacen los docentes respecto de cuáles son los aprendizajes más significativos realizados por los estudiantes. En general en todas las entrevistas se alude a dos cuestiones fundamentales: (a) la transformación personal que se produce en los estudiantes al implicarse en contextos sociales de mayor vulnerabilidad a los idealizados para el desarrollo de la profesión. (e.g., "pibes que han hecho cosas que los impactan, que los cambian, que los reorienta en la profesión. Y otros que pasaron por las prácticas, cumplieron requisito”). (b) La re-significación desde una perspectiva crítica de los saberes en la práctica concreta en una organización (e.g., "el darse cuenta de las herramientas que fue aprendiendo en la facultad y poder aplicarlas a ese tipo de organización diferente”).

\section{Discusión}

¿El espacio de PSC es capaz de producir transformaciones cualitativas en la formación de los estudiantes? ¿Es el APS la herramienta pedagógica pertinente para adecuarse a los desafíos de la Educación superior en la actualidad? ¿Se hace efectivo el cumplimiento de la responsabilidad social universitaria por medio de la implementación de las PSC?

Cecchi \& Pérez (2013), señalan que las PSC promueven transformaciones en las dimensiones ética, epistemológica, social y pedagógica. Nos proponemos vincular los resultados obtenidos en la presente investigación con estas dimensiones de análisis.

1. Dimensión Ética

Esta dimensión se asocia con la aparición de conductas vinculadas con el debate y reflexión acerca del modelo de Universidad vigente, su pertinencia (Muñoz, 2008), la responsabilidad social y ética de la misma, y el compromiso comunitario (Mazzetti, 2014). Se vincula fundamentalmente con el desarrollo de valores como la solidaridad, el altruismo, el respeto por el otro, la tolerancia con lo diferente, entre otros.

Encontramos evidencia de la presencia de esta dimensión en categorías como "Sentimientos positivos hacia sí mismos / Autosuperación”, de la dimensión "Reacciones", en "Competencia participativa" en la dimensión de Aprendizaje, y en "Compromiso ético del rol" en el nivel de Transferencia. (e.g., "Al cursar el seminario y realizar las prácticas pudimos dimensionar otras funciones de la Universidad, que muchas veces se encuentran relegadas. Comprendimos que la formación tiene que abarcar diferentes aspectos, no sólo el académico o profesional. Tiene que ir más allá, insertarse en el ámbito, preocuparse por el contexto, generar compromiso y propugnar por el cambio. Es una práctica basada en valores (como solidaridad, fraternidad, e igualdad). Al hacer la práctica no sólo transformamos la comunidad, sino también nuestra Facultad, cumpliendo con su misión social.")

Estos resultados son consistentes con los objetivos planteados para el ejercicio de la Responsabilidad Social Universitaria por De la Cuesta González, de la Cruz Ayuso y Fernández (2010), entendida como la capacidad de la universidad de promocionar y ejercer un 
conjunto de principios y valores por medio de procesos y prácticas en sus cuatro funciones capitales: investigación, docencia, extensión y gestión. Las evaluaciones que realizan los propios estudiantes sobre su experiencia en la práctica articulan adecuadamente con lo deseado para la formación de profesionales socialmente comprometidos.

\section{Dimensión Epistemológica}

Las PSC constituyen una posibilidad de transferir conocimientos a la comunidad colaborando en la resolución conjunta de problemáticas concretas. Esta intervención posibilita un enriquecimiento de ambas partes, contribuyendo a reconsiderar nuevas líneas de investigación. Permite advertir la transformación que el conocimiento puede producir en la comunidad. Este posicionamiento respecto a la reciprocidad del aprendizaje se sustenta en la utilización de la metodología de Aprendizaje Servicio (Tapia, 2002)

Se citan a continuación fragmentos del discurso de los estudiantes evaluados en categorías de todos los niveles de análisis.

El análisis de los resultados pone en evidencia que el Seminario de Prácticas Socio Comunitarias responde efectivamente a la metodología de Aprendizaje Servicio, ya que se observa un equilibrio adecuado entre los procesos de Aprendizaje por un lado y de Servicio por el otro. Luego de relevados todos los datos, el $82 \%$ de los estudiantes realizaron informes donde dan cuenta de la reciprocidad del aprendizaje entre ellos y las organizaciones, y de la existencia de un servicio concreto brindado a la comunidad. (e.g., "Nuestro rol fue activo y consideramos necesario que puedan llevarse a cabo estas prácticas en todas las facultades ya que no solo brindamos nuestro conocimiento, sino también, la comunidad nos dio el suyo”.

"Creemos importante la implementación del Seminario en toda la Universidad. El conocimiento aprendido en la facultad tiene que ser puesto a disposición de la comunidad y nosotros recibir el de ellos”.)

En el Aprendizaje Servicio. Tanto el aprendizaje como el servicio comunitario son privilegiados enfáticamente, porque está necesariamente protagonizado por los estudiantes, tiene una intencionalidad solidaria y otra pedagógica, proponiéndose mejorar la calidad de los aprendizajes escolares.

\section{Dimensión social}

Cuando la Universidad se permite dialogar y discutir sobre su misión comunitaria con todos los actores sociales, se dispone a aprender y transformarse. Promueve la inclusión, sentimientos de pertenencia, desarrollo de habilidades sociales, participación ciudadana, y habilidades de carácter político para la gestión de la convivencia en sociedad.

Encontramos evidencia de transformaciones en esta dimensión en categorías como "Sentimientos de comunidad”, "Sentimientos positivos hacia sí mismos / Autosuperación”, y "Resultados / Objetivos cumplidos" del nivel de Reacciones, en las categorías "Competencia Social” y "Competencia participativa” en el nivel de Aprendizaje. (e.g., "Más allá de los objetivos alcanzados fuimos parte de la comunidad, contribuimos desde un pequeño lugar a la sociedad. Aprendimos muchas cosas que quedan fuera del aprendizaje dentro de las aulas, pero que son de vital importancia a la hora de desarrollo profesional. Ya sea el trato con las personas, la resolución de problemas al margen de nuestra profesión, conocer otras realidades, ser más humanos y solidarios, mirar más allá de nuestros propios problemas.")

Las respuestas de los estudiantes han evidenciado que las PSC son consistentes con la misión de la Educación Superior (la Declaración de la Conferencia Mundial sobre la Educación 
Superior del año 2009 (p.1),), y que la pertinencia social (M. Muñoz, 2008), está asegurada, promoviendo acciones concretas de servicio comunitario.

Gasca-Pliego y Olvera-García (2011), por su parte, sostienen que la universidad debe crear nuevas estrategias pedagógicas para el desarrollo de competencias sociales y participativas que incrementan los conocimientos y habilidades de los estudiantes para desenvolverse profesionalmente en la vida social.

3. Dimensión Pedagógica

Las prácticas posibilitan una resignificaciónpor medio de la reflexión de los conceptos teóricos y de una experiencia de aprendizaje en territorio, y cuyo beneficio radica en que permite que los estudiantes resignifiquen los contenidos de aprendizaje frente a problemas concretos, participen activamente y con autonomía en la construcción del conocimiento y desarrollen habilidades críticas y reflexivas.

A continuación se transcriben citas de los propios estudiantes que dan cuenta de transformaciones en las categorías de "Competencia Metodológica" e "Insuficiente formación académica" en el nivel de Aprendizaje, así como en "Polivalencia del rol" e "Incumbencias profesionales" en el nivel de Transferencia. (e.g., "Otro aspecto para desatacar es haber aprendido a trabajar con este tipo de asociaciones sin fines de lucro, dado que en la facultad nos preparan principalmente para brindar servicios en organizaciones con fines de lucro y gracias a estas prácticas uno puede tomar contacto con otros sectores de la sociedad que requieren de ayuda profesional.”

"Lo aprendido en las prácticas nos sirvió para bajar a la realidad nuestro conocimiento y aprender, a la vez, aquellas cuestiones que desconocíamos, como trámites, leyes nuevas, etc.”)

Los resultados dan cuenta de la capacidad de las PSC, a partir de la propuesta pedagógica de APS, para promover en los estudiantes una reflexión crítica acerca de los contenidos académicos, evaluando su pertinencia, alcances y limitaciones. Esto conlleva una trasformación y resignificaciónde los contenidos teóricos y técnicos adquiridos por los estudiantes en su proceso de formación.

\section{Conclusiones}

En esta investigación se logró una aproximación exploratoria, descriptiva y cualitativa respecto del compromiso social y la potencialidad transformadora de las PSC de una institución pública de educación superior.

El método de análisis cualitativo permitió dar cuenta, con el propio discurso de los actores sociales implicados, de cuáles son las significaciones atribuidas a este espacio de formación y los aprendizajes asociados. Para sistematizar estos resultados se utilizó el modelo de evaluación de acciones formativas de Kirkpatrick (1999) que permitió describir los resultados en cuatro niveles de análisis: Reacciones, Aprendizaje, Transferencia e Impacto Organizacional.

Los resultados dan cuenta de un 95\% de satisfacción (nivel Reacciones) de los estudiantes con la formación recibida atribuyendo esta valoración a sentimientos de autosuperación aparecidos con motivo de esta práctica y al refuerzo de sentimientos comunitarios, desarrollo de la solidaridad y pertenencia social. Por otra parte, los estudiantes dieron cuenta del desarrollo de las competencias metodológicas, participativas y sociales según el modelo de 
competencias de Bunk (1994), evidenciando que los aprendizajes no sólo mejoran su formación académica, sino su empleabilidad futura.

Los docentes valoraron satisfactoriamente el Aprendizaje realizado por los estudiantes que se implican en su práctica, ya sea mediante la resignificación desde una perspectiva crítica de los saberes en la práctica concreta, o bien por la transformación personal que se produce en ellos al implicarse en contextos sociales de mayor vulnerabilidad a los idealizados para el desarrollo de la profesión.

Con relación al impacto que las PSC tuvieron en el nivel de Transferencia, las respuestas se distribuyeron en el desarrollo de la Polivalencia del rol, definida como la diversidad de valencias que adquiere el rol profesional; Aprendizaje de las Incumbencias profesionales; resignificación del Compromiso ético del rol, referido a la valoración de los aspectos éticos del ejercicio del rol profesional.

El último nivel, de Impacto Organizacional arrojó resultados que incluyeron impactos positivos en el 90,4\% de las respuestas, distribuidas en varias categorías: impactos en las personas, los procesos, los resultados y en la propia universidad.

Los resultados permiten concluir que la realización de las PSC, a través de la metodología de APS, promueve la transformación de los estudiantes en diversas dimensiones: epistemológica, social, pedagógica y ética. Estas transformaciones promueven la aparición de conductas prosociales que favorecen el desarrollo ciudadano, el ejercicio responsable del rol profesional, el debate crítico sobre los valores éticos en juego en el quehacer universitario, la reflexión sobre las necesidad de transformar la formación académica para que se adapte a las demandas de la sociedad actual, y la posibilidad de que los estudiantes puedan pensarse como futuros actores sociales comprometidos con el devenir de la comunidad.

El análisis realizado sobre estas PSC nos permite concluir que su implementación es consistente con los nuevos desafíos que debe enfrentar la educación superior del siglo XXI, creando nuevas estrategias pedagógicas centradas en la conformación de comunidades de aprendizaje con foco en gestión de problemas y proyectos sociales, así como la promoción de la interdisciplinariedad. Definir estas prioridades es de vital importancia y constituye un compromiso ético ineludible, y valoramos que esta experiencia de PSC, con su implementación, contribuya a construir este modelo de universidad inclusiva, solidaria y democrática.

\section{Referencias}

Almenara, J. C., \& Abril, F. L. (2002). Elaboración de un sistema categorial de análisis de contenido para analizar la imagen del profesor y la enseñanza en la prensa. La Investigación en los Medios de Comunicación, 1-25.

Bunk, G.P. (1994). La transmisión de competencias en la formación y perfeccionamiento profesionales de la RFA. Revista Europea de Formación Profesional, 1, 8-14.

Campo, L. (2008). El aprendizaje servicio en la universidad como propuesta pedagógica. En M. Martínez (Ed.), Aprendizaje servicio y responsabilidad social de las universidades (pp. 81-91). Barcelona: Octaedro-ICE.

Cecchi, N., Delicio, F. A., Governatori, V., Libera, M. E., Puglisi, S., \& Zaballa, E. (2010, diciembre). Prácticas profesionales comunitarias en la Facultad de Cs. Económicas y 
Sociales de la Universidad Nacional de Mar del Plata. Presentada en el $X$ Coloquio Internacional sobre Gestión Universitaria en América del Sur, Buenos Aires, Argentina

Cecchi, N. H., \& Pérez, D. A. (2013, septiembre) Enseñar y aprender “en” y “de” la comunidad: Aproximación conceptual sobre el valor de las prácticas Socio Comunitarias. Presentada en Jornadas Nacionales sobre la Formación del Profesorado, Buenos Aires, Argentina.

Conferencia Mundial sobre la Educación Superior (2009). La nueva dinámica de la Educación Superior y la investigación para el cambio social y el desarrollo, Comunicado de Prensa. Recuperado el 18 de mayo de 2010, de: http://www.unesco.org/education/WCHE2009/comunicado_es.pdf

de la Cuesta González, M., de la Cruz Ayuso, C., \& Fernández, J. M. R. (2010). Responsabilidad social universitaria. (pp. 189-190). La Coruña: Netbiblo.

Flanagan, J. C. (1954). The critical incident technique. Psychological Bulletin, 51(4), 327-358.

García Guadilla, C. (2008). El compromiso social de las universidades. Cuadernos del Cendes, 25(67), 129-134.

Gasca-Pliego, E., y Olvera-García, J. C. (2011). Construir ciudadanía desde las universidades, responsabilidad social universitaria y desafíos ante el siglo XXI. Convergencia, 18(56), 37-58.

Guerra- López, I. (2007). Evaluación y mejora continua. Conceptos y herramientas para la medición y mejora del desempeño. Indiana: AuthorHouse.

Kirkpatrick, D. L. (1999). Evaluación de acciones formativas. Los cuatro niveles. Barcelona: EPISE.

Lara, R., y Bris, M. (2010). La responsabilidad social en la gestión de centros universitarios. Revista Ibero-Americana de Estudos em Educação, 5(2), 1-15.

Mazzetti, S. (2014), Responsabilidad Social Universitaria o Compromiso Social Universitario. En M.I., Pacenza, y Y.F. Silva Peralta (Coord), Educación Superior y Voluntariado (pp. 57-70). Mar del Plata: Grupo SOVIUC.

Muñoz, M. (2008). Pertinencia y nuevos roles de la educación superior en la región. En C. Tünnermann Bernheim (Ed.), La educación superior en América Latina y el Caribe: diez años después de la Conferencia Mundial de 1998 (pp. 223-266). Cali: Sello Editorial Javeriano-Iesalc-UNESCO.

Navarro, P., \& Díaz, M. (1994). Análisis de contenido. En J. Delgado, y J. Gutierrez (Coord.), Métodos y técnicas de cualitativas de investigación en ciencias sociales (pp.177-224). Madrid: Síntesis Psicología.

Navarro, R., López, A., \& Barroso, P. (1998). El análisis de incidentes críticos en la formación inicial de maestros. Revista Electrónica Interuniversitaria de Formación del Profesorado, 1(1). Recuperado el 24 de Noviembre de 2011 desde: http://aufop.com/aufop/uploaded_files/articulos/1224541140.pdf

Nail, O. (2010). Los incidentes críticos de aula. Un aporte a la gestión docente y la formación inicial. Proyecto DIUC 210.161.006-1.0. Concepción: Universidad de Concepción.

Olea Soto, C. (2010). Evaluación de impacto de la capacitación: Caracterización y una propuesta para la gran empresa privada chilena. Memoria Teórica para optar al Título de Psicóloga, Facultad de Ciencias Sociales, Universidad de Chile. 
Rothwell, W., Hohne, C., \& King, S. (2007). Human performance improvemente. Building practitioner competence. Oxford: Elsevier Inc.

Rutty, M. G. (2007). Evaluación de Impacto en la Capacitación de Recursos Humanos. Tesis Doctoral, Facultad de Ciencias Económicas, Universidad de Buenos Aires.

Schvarstein, L. (2003). La inteligencia social de las organizaciones. En M. Torres Pernalete, y M. Trápaga Ortega (Eds.), Responsabilidad Social Universitaria. Retos y Perspectivas. Buenos Aires: Paidós.

Tapia, M. N. (2010). La propuesta pedagógica del "Aprendizaje-Servicio": una perspectiva latinoamericana. Tzhoecoen, revista científica, 0(5), 23-43.

Tripp, D. (1993). Critical incidents in teaching: Developing professional judgment. Nueva York: Routledge. 